\title{
ARTÍCULOS
}

\section{Evaluación formativa: un reto para el docente en la educación a distancia}

\author{
Formative assessment: a challenge for teachers in distance \\ education
}

\author{
García Riveros, Janet Meluzka; Farfán Pimentel, Johnny Félix; Fuertes \\ Meza, Luis Carmelo; Montellanos Solís, Amparo Rosa
}

\author{
Janet Meluzka García Riveros \\ jamega20@gmail.com \\ Universidad César Vallejo, Perú \\ (D) Johnny Félix Farfán Pimentel \\ jfarfanp@ucv.edu.pe \\ Universidad César Vallejo, Perú \\ (D) Luis Carmelo Fuertes Meza \\ lfuertesmeza@hotmail.com \\ Universidad César Vallejo, Perú
}

Amparo Rosa Montellanos Solís amparomontellanos19@gmail.com

Universidad César Vallejo, Cuba

\section{Delectus}

Instituto Nacional de Investigación y Capacitación Continua, Perú ISSN-e: 2663-1148

Periodicidad: Semestral

núm. 2, 2021

publicaciones.iniccperu@gmail.com

Recepción: 22 Mayo 2021

Aprobación: 11 Junio 2021

Publicación: 01 Julio 2021

Esta licencia permite a otros entremezclar, ajustar y construir a partir de su obra con fines no comerciales, y aunque en sus nuevas creaciones deban reconocerle su autoría y no puedan ser utilizadas de manera comercial, no tienen que estar bajo una licencia con los mismos términos. \section{NoComercial 4.0 Internacional.}

Esta obra está bajo una Licencia Creative Commons Atribución-
Resumen: El presente artículo tiene por objetivo realizar una revisión y análisis crítico de la bibliografía más relevante sobre la evaluación formativa en educación y sus implicancias. En este contexto a distancia, a causa del estado de emergencia sanitaria, basado en la problemática que significa evaluar actualmente en todo el nivel educativo; y las limitaciones que enfrenta de manera cotidiana el proceso de enseñanza en las aulas de forma presencial y ahora que se brinda en espacios educativos virtuales, se agudiza la problemática considerando inconsistencias en el manejo de las teorías sobre evaluación, la cual se pretende actualizar con respecto a la evaluación formativa y la retroalimentación para mejorar los resultados de aprendizaje. En la primera parte de este artículo, se brinda una visión teórica de la evaluación formativa y su importancia para el proceso educativo actual. En la segunda parte, se analiza las limitaciones de la aplicación de la evaluación formativa en la educación a distancia y sus implicancias para el desarrollo de los aprendizajes, se incluye los elementos más importantes que dan cuenta del ámbito de la evaluación del aprendizaje. Luego, se considera la metodología empleada que se centró en la investigación documental de artículos científicos de los 6 últimos años en revistas indexadas. Se concluye lo importante que es realizar un análisis de los resultados de la revisión sistemática, asimismo desarrollar una discusión entre la información más relevante de la bibliografía considerada. Para finalizar, se brinda las conclusiones basadas en el desarrollo de la investigación.

Palabras clave: evaluación formativa, contexto a distancia, retroalimentación.

Abstract: The purpose of this article is to carry out a review and critical analysis of the most relevant bibliography on formative evaluation in education and its implications. In this distance context, due to the state of health emergency, based on the problematic that means to evaluate currently in all the educational level; and the limitations faced daily by the teaching process in the classroom and now that it is provided in virtual educational spaces, the problem is aggravated considering inconsistencies in the management of the theories on evaluation, which is intended to be updated with respect to formative evaluation and feedback to improve learning outcomes. In the first part of this article, a theoretical vision of formative 


\begin{abstract}
assessment and its importance for the current educational process is provided. In the second part, the limitations of the application of formative assessment in distance education and its implications for the development of learning are analyzed, including the most important elements that account for the scope of learning assessment. $\square$ en, the methodology used is considered, which focused on the documentary research of scientific articles of the last 6 years in indexed journals. It is concluded how important it is to analyze the results of the systematic review and to develop a discussion among the most relevant information of the considered bibliography. Finally, conclusions are drawn based on the development of the research.
\end{abstract}

Keywords: formative evaluation, distance context, feedback.

\title{
INTRODUCCIÓN
}

En el contexto mundial actual, los requerimientos educativos para brindar aprendizajes de calidad se centran en la evaluación formativa que es una necesidad para valorar los procesos de aprendizaje en el desarrollo de competencias (Fraile et al., 2019). En vista a lo antes mencionado, es primordial reconsiderar la forma de evaluar porque en estos tiempos constituye uno de los problemas más complejos de la práctica pedagógica en el aula, en vista que la mayoría de veces es únicamente cuantitativa, es así, que la forma de evaluar debe presentar un cambio para relacionarse al logro de los aprendizajes más allá del elemental resultado numérico (Andrade \& Brookhart, 2019). En suma, considerando que los educadores deben asumir compromisos para evolucionar en sus prácticas de valoración para el aprendizaje.

Respecto al ámbito latinoamericano, la problemática de la evaluación se refiere al limitado uso y manejo de los instrumentos de evaluación de carácter formativo por parte de los maestros (Pérez et al., 2019). Cabe agregar, que en Chile las prácticas educativas de evaluación persisten en el enfoque tradicional en el cual predomina el aprendizaje memorístico, por lo cual, es momento de que los profesores reformulen su visión a los procesos de evaluación teniendo siempre presente las características, necesidades e intereses de los estudiantes (Serra et al., 2018). De este modo, poder lograr niveles reales de aprendizaje que nos permita realizar propuestas de mejora. A partir de este razonamiento, se apuesta por el desarrollo de una evaluación por competencias que enmarque un proceso pedagógico integral (Morales \& Medina, 2020). De este modo, debemos reflexionar sobre la práctica evaluativa, dando prioridad a la función formativa en el proceso de enseñanza-aprendizaje.

Frente a ello, es necesario concebir a la evaluación formativa como el proceso cíclico mediante el cual los estudiantes y sus docentes realizan el seguimiento, recopilación y el procesamiento de la información para poder llegar a resultados que permitan emitir juicios y realizar una toma de decisiones sobre el aprendizaje. La información obtenida se puede utilizar como retroalimentación para que cada estudiante active los procesos internos y autorregule sus aprendizajes (Black \& William, 1998 citado por Valdivia \& Fernández, 2020). Asimismo, la evaluación formativa resulta una actividad continua que se desarrolla durante todo el proceso educativo con la finalidad de regularlo; por tanto, valorar los resultados no es lo más importante, sino comprender el proceso, controlarlo e identificar las posibles dificultades y en qué medida estas pueden corregirse con nuevas propuestas de las actividades formativas y de las estrategias previstas en beneficio del aprendizaje del estudiante (Cunill \& Curbelo, 2021).

Respecto a la evaluación formativa pretende desarrollar un aprendizaje responsable y consciente en el 
estudiante mediante el acompañamiento del docente que permita construir una estructura del aprendizaje de forma sistemática; es así, que las escuelas deben propiciar propuestas innovadoras de aprendizaje con herramientas y recursos pertinentes para tal fin. En este contexto, es fundamental tener claridad que la evaluación formativa se caracteriza por utilizar diferentes técnicas, medios e instrumentos entre los cuales están; la observación, la lista de cotejo, las rúbricas, la entrevista, el portafolio, los diferentes tipos de organizadores, el registro de desempeño y otros que sean funcionales para los actores educativos (Segura, 2018).

Por otra parte, es importante señalar que las fases de la evaluación formativa son motivar y retroalimentar que consiste en identificar los espacios de aprendizaje en que se encuentra el estudiante desde su punto de partida hasta el punto al que se desea llegar para eliminar la brecha de aprendizaje (Black \& William, 1998 citado en Bizarro et al., 2019). Asimismo, se debe tener presente que los procedimientos para la evaluación formativa consideran tres dispositivos metodológicos por la importancia que tienen en cuanto a la frecuencia de su empleo en las prácticas educativas en el aula: el interrogatorio, la retroalimentación mediante las calificaciones y la coevaluación y autoevaluación (Moreno, 2016).

Además, es necesario señalar, que la evaluación formativa tiene como base el paradigma constructivista que se basa en la búsqueda de evidencias de una manera intencionada, involucrada y comprometida a los estudiantes en la gestión de su propio aprendizaje a partir de procesos metacognitivos y reflexivos (Minedu, 2019). Agregando al respecto, los cambios de la evaluación formativa se deben principalmente a la influencia de las teorías constructivista, cognitiva y sociocultural del aprendizaje en donde el estudiante regula su propio aprendizaje y se resaltan aspectos claves, como lo son la planificación de objetivos de aprendizaje; la retroalimentación continua que incluye autoevaluación, coevaluación y evaluación de pares (Medina \& Deroncele, 2019).

Por otra parte, se destaca que la valoración de los niveles de logro debe tener en cuenta el proceso sistemático, sistémico, participativo y reflexivo (Pasek de Pinto \& Mejía, 2017). Para poder desarrollar la evaluación formativa es necesario tener en cuenta la importancia de la retroalimentación como estrategia, pero esta vez orientada entre pares, es decir, entre los compañeros del aula, la cual se define como la información que recibe un alumno a través de un compañero sobre las tareas relacionadas que realiza para mejorar su rendimiento académico (Huisman et al, 2019). Además, la evaluación congrega el centro de todo el proceso educativo mediante las interacciones que se generen en el aula, partiendo de la experiencias y actividades propuestas para en momentos oportunos realizar la retroalimentación, lo cual verifica el nivel de aprendizaje logrado (Rodríguez \& Salinas, 2020).

Asimismo, es de suma importancia el involucramiento emocional de los estudiantes, que se expresa de manifiesto en base a la retroalimentación, durante la reflexión de sus propias autoevaluaciones (Leighton, 2019). Cabe señalar que el docente es el que debe guiar el proceso interpretativo del estudiante promoviendo las capacidades de orden superior de carácter crítico y reflexivo (Pellegrino, 2020). Además, la retroalimentación es formativa porque mejora la habilidad y recomiendan que la interacción académica sea en equipo estudiantil; ya que así se desarrolla mayor compromiso para la mejora del aprendizaje (Huisman et al, 2019). Sin embargo, hay poca investigación psicológica sistematizada sobre los estudiantes y las interpretaciones de la retroalimentación que hacen a través de modelos mentales (Leighton, 2019).

En la actualidad, quizás el futuro puede parecer incierto sobre todo en esta época de pandemia (Díaz, 2020). Sin embargo, para que podamos tener control sobre el futuro de la educación, debemos hacer serias transformaciones en el sistema de evaluación partiendo de la retroalimentación constante con nuestros estudiantes y usándola como estrategia para mejorar el desarrollo del pensamiento complejo a través de la educación a distancia (Medina \& Deroncele, 2020). Al respecto, es necesario entender que la evaluación se puede realizar sin incluir necesariamente una calificación cuantitativa; de hecho, puede tener mayor impacto y ser más motivada para el estudiante una evaluación formativa y permanente (López, 
et al., 2019).

Cabe señalar que evaluando de manera pertinente se garantiza la valoración de los avances en el proceso de aprendizaje de cada estudiante, es así que al desarrollar las competencias requeridas siempre acompañados por su docente serán capaces de identificar y reflexionar sobre la problemática de su comunidad y proponer

acciones innovadoras de mejora que contribuirán en la solución de problemas y transformar su realidad (Buenrostro, 2016). En ese sentido la evaluación formativa es importante porque permite mostrar a los estudiantes dónde se encuentran en el proceso de aprendizaje y hacia donde pueden llegar, así como ayudarles a progresar tomando conciencia de cómo hacerlo, con el convencimiento de que el aprendizaje y la evaluación son inseparables en la medida que favorecen el proceso de aprendizaje constructivo, reflexivo y eficaz (López, 2017).

Por otra parte, se debe tener en consideración que la evaluación formativa logra que los estudiantes sean activos, participativos con sus compañeros y maestros; ellos comparten sus propósitos de aprendizaje y comprenden cómo van progresando, que es lo que necesitan aportar, como hacerlo y cuando hacerlo para mejorar, en este proceso los estudiantes desarrollan sus habilidades de autoevaluación y coevaluación (Heritage, 2007 citado por Moreno 2016). En consecuencia, evaluar de manera formativa es lograr que los estudiantes sean autónomos en la gestión de sus propios aprendizajes al tomar conciencia de sus debilidades, dificultades, necesidades, intereses y fortalezas en el cual van a incrementar la confianza y seguridad en sí mismo. Ante la perspectiva actual desde el inicio de la pandemia, muchos gobiernos se vieron obligados a crear y proponer procesos educativos que respondan a las necesidades de la denominada nueva normalidad, que fueron implementados considerando los diferentes entornos virtuales y accesibilidad de cada realidad.

A partir de lo acontecido se implementó la educación a distancia que se presenta como reto para los actores educativos al cambiar el proceso de enseñanza y aprendizaje a una forma carente de contacto directo y personalizado, lo cual pone en riesgo la calidad del aprendizaje (Intriago, et al., 2020). Una preocupación resaltante en este contexto de educación a distancia, es que la evaluación en línea ofrece una tendencia hacia formas tradicionales de evaluación (exámenes vigilados y tareas escritas), así como pocas oportunidades para la variedad en las evaluaciones y limitado desarrollo de habilidades genéricas tales como habilidades comunicacionales, capacidades tecnológicas para la lectura y escritura, solución de problemas, trabajo en equipo y otras similares (Dorrego, 2016).

A partir de esta realidad, se debe plantear que ventajas podríamos aprovechar de este proceso educativo virtual, que fue considerado por muchos años en el desarrollo educativo, pero se limitó su real importancia. Cabe señalar, que la educación virtual en las escuelas fue considerada solo como un apoyo, y esto en el caso de contar con recursos para su implementación, ahora el panorama cambió convirtiéndose en una oportunidad para continuar con el aprendizaje escolar (Barrón, 2020). Por otra parte, se sugiere que la evaluación a distancia inicie por la autoevaluación, es decir que los estudiantes sean los protagonistas responsables de la valoración de sus propios aprendizajes; la autoevaluación es la base fundamental por la cual se recoge, sistematiza y unifica la información con el propósito de que la institución educativa reflexione sobre sus debilidades y fortalezas para mejorarlas con propuestas creativas y realistas (Bañuelos \& Montero, 2017).

Asimismo, debemos tener en cuenta que al hacer uso de la tecnología de la información y comunicación los docentes tienen que tener claro los fundamentos pedagógicos de aprendizaje y evaluación a la vez observar que aspectos mejoran o delimitan los logros de estos procesos para que se implementen otras estrategias de estudio o enseñanza (Lezcano \& Vilanova, 017).

Para finalizar, luego del análisis de la literatura realizada con respecto a la evaluación, evaluación formativa, retroalimentación y su implicancia en la educación a distancia. Es primordial llegar a una reflexión que permita la apertura de los campos de acción pedagógicos para mejorar las prácticas de evaluación 
formativa y más aún ahora en este contexto de educación a distancia donde el reto docente es adecuar las estrategias ya existentes e innovar otras que se adecuen a la realidad de los educandos. Es por ello, que en este artículo se considera como objetivo el análisis y reflexión de la información actualizada y relevante relacionada a la evaluación formativa en el contexto actual de educación a distancia como propuesta teórica que amplíe el panorama a los agentes educativos para reconsiderar el servicio educativo que se brinda en las escuelas.

\section{METODOLOGÍA}

Se realizará una revisión de la literatura descriptiva con el fin de ampliar el panorama teórico de la evaluación formativa en el contexto actual de las escuelas que constituye un reto para el docente en su desempeño cotidiano. Según Vera (2016), la revisión descriptiva actualiza al lector sobre conceptos útiles en áreas en permanente evolución. Cabe señalar que tiene una gran utilidad en la enseñanza, y también interesará a muchas personas de otros campos afines. Se consideró para tal efecto el método análisis-síntesis, que nos permite realizar un análisis de los argumentos científicas encontrados y sintetizar la información relevante (Deroncele et al., 2020).

Cabe señalar que el análisis epistemológico se basa en 34 referencias "open access" encontradas en Scopus, Ebsco, Scielo, Proquest, Dialnet con el término de búsqueda o descriptor utilizando solo el título del articulo y con restricción en el tiempo considerando los últimos 6 años. También, se empleó como principal método el análisis documental teniendo en cuenta que el principal objetivo radicó en la sistematización de la información encontrada. El estado del arte se plantea como una línea metodológica sobre la base de dos pilares constituyentes: la heurística y la hermenéutica (Londoño, et al., 2014, citado en Medina \& Deroncele, 2019, p. 599).

\section{RESULTADOS}

La evaluación es una pieza clave para producir información necesaria a lo largo de todo este proceso, forjando en el estudiante la identificación de sus fortalezas, oportunidades de mejora y sobre todo de sus potencialidades formativas (Deroncele et al., 2020). Desde el entorno virtual, es determinante, concebir un ambiente de aprendizaje, aún más poderoso y capaz de producir una revolución en la educación (Díaz, 2020). De esta manera, se plantea un punto de partida histórico lógico, que ayude a entender cómo se imprime este concepto en el actual fenómeno educativo y como ello representa una oportunidad de evolución y de transformación social (Villalaz \& Medina, 2020).

En la educación, se utiliza el Internet y empiezan a surgir los entornos virtuales como opciones educativas, algunos de ellos como el Massive Open Online Course (MOOC). A partir de este momento, habría que plantearnos, que las oportunidades de asumir un proceso educativo on-line, siempre tuvieron un espacio y tiempo histórico suficiente. Sin embargo, ello no fue aprovechado o valorado en su momento.

Por mucho tiempo, la educación virtual no representó una prioridad pedagógica, hasta el momento de la pandemia (Barrón, 2020). Para entonces, todo fue vertiginoso y exigió que, en automático, los actores y las instituciones incorporasen de manera urgente, una evaluación remota. Para originar una verdadera revolución desde la educación, es necesario adaptar el currículo tomando en cuenta las habilidades del siglo XXI, como la innovación, el manejo de medios de información y el uso de TIC; y reinventar el sistema evaluativo, de tal forma que responda a una pedagogía integral y de orientación formativa (Morales \& Medina, 2020).

Si se quiere llevar el concepto de evaluación formativa a la educación no presencial y remota, el desafío se vuelve aún más difícil, pero no es tarde para idear cómo hacerlo en una realidad como la peruana. 
Incluso, hay posturas que integran tres propuestas: evaluación del aprendizaje, la evaluación para el aprendizaje y el aprendizaje para toda la vida (Moreno, 2016; Medina \& Deroncele, 2020b). Cuando el alumno ve logros alcanzados de manera real, se motiva para poder seguir aprendiendo. Para ello, se debe lograr evaluaciones tanto presenciales como virtuales más eficientes que ayuden a que los alumnos puedan ir controlando su propio éxito.

Al respecto, la evaluación del aprendizaje que es concepto básico de este análisis tiene como principal objetivo medir el alcance de las metas de aprendizaje que se presentan en el currículo tanto de manera cuantitativa como cualitativa. Se asume con ello, alentar un cambio permanente sobre el conocimiento de los estudiantes a través del desarrollo de competencias. Dicho tipo de evaluación sería eficaz a través del método o procedimiento que implemente el docente en el aula relacionando coherentemente los indicadores de evaluación con las metas de aprendizaje.

\section{DISCUSIÓN}

Los actores educativos y de manera particular los docentes que son quienes facilitan el aprendizaje demuestran una gran predisposición para asimilar y adecuarse a los cambios que conlleva las dificultades para desarrollar los procesos educativos actuales que son muchas veces desconocidos porque no fueron formados para enseñar a distancia y menos para evaluar (Díaz, 2020). Cabe señalar que la teoría educativa puede reformularse constantemente en un sistema que es caótico por naturaleza en donde, a saber, de Medina \& Deroncele $(2019 ; 2020$ b), el docente debe buscar la simplicidad dentro de la complejidad definiendo su perfil docente.

La capacidad de adaptación y reinvención de millones de docentes en el mundo ha quedado demostrada ante el acelerado proceso de digitalización que ha sufrido la educación presencial, llevada ahora a un entorno de totalidad virtual. Por efecto de este sistema tan caótico producido por la pandemia se ha logrado un orden dentro del caos (Barrón, 2020). Se ha dado paso a la incertidumbre en donde la creatividad se ha impuesto para lograr una lógica dentro de las nuevas metodologías educativas inauguradas por los docentes en un ambiente de aprendizaje virtual (Díaz, 2020).

Sin embargo, como ya se dijo anteriormente, seguramente no tenemos aún el perfil de docente ideal en el Perú para desarrollar una evaluación formativa en este nuevo sistema educativo virtual. El perfil docente debe desarrollar un accionar político para que solucione problemas en su ejercicio profesional convirtiéndose en el líder transformador que la sociedad necesita (Villalaz \& Medina, 2020). La escuela debe desarrollar un pensamiento crítico y reflexivo para poder solucionar los problemas que el país necesita (Medina \& Deroncele, 2020a), no solo desde Lima, la capital del país, sino desde las zonas rurales. Sin embargo, a nivel país no se tiene aún una ruta clara de cómo ir subiendo peldaños y, por ende, cómo configurar una cultura evaluativa que contemple dichos aspectos (Morales \& Medina, 2020).

En el informe del estado de políticas públicas docentes (Cuenca \& Vargas, 2018), comprendido en el período 2012-2017, se establece una reforma educativa que logre el bienestar docente centrándose en preparar el campo para una enseñanza efectiva, atraer y retener talento y gestionar un buen desempeño. Si bien hay intentos de mejorar este hecho, aún tenemos un déficit en los rangos salariales y se denota una falta de integración entre los grupos de maestros dentro de las instituciones en las que laboran: individualismo en vez de trabajo interdisciplinario y transdisciplinario.

Este desafío pueda alcanzarse trabajando lo que OCDE (2017) propone sobre los ambientes innovadores de aprendizaje, cuyos constituyentes principales son la tecnología, un aprendizaje continuo y autodirigido, una alianza entre el hogar y la escuela, la investigación y una evaluación formativa integral que responda las siguientes preguntas: ¿dónde están nuestros alumnos?, ¿hacia dónde los llevamos?, ¿qué necesitamos para llevarlos allí? Por consiguiente, este segundo desafío se obtiene con un docente desde su rol reflexivo que 
debe revisar: ¿cuál es su propósito en la enseñanza?, ¿cómo lo ha previsto? y ¿qué necesita ser reajustado en este proceso?; sin esperar el momento final del aprendizaje. La reflexión permite esta disposición de flexibilidad porque hace que el docente haga un alto en su "saber hacer" para enfocar su "ser pedagógico". Es decir, debe comprender quién es el estudiante y qué necesita para aprender (Medina \& Deroncele, 2020b). Es un desafío que también implica el proceso formativo y adaptativo del docente; ya que el gran reto que tiene es repensar la educación en el proceso de enseñanza desde una perspectiva compleja y transdisciplinar; es decir, a través de las diferentes disciplinas y más allá de toda disciplina enseñándole al estudiante cómo comprender el mundo de una mejor manera (Medina \& Deroncele, 2019).

El futuro puede parecer incierto sobre todo en esta época de pandemia en la que vivimos (Díaz, 2020). Sin embargo, para que podamos tener control sobre el futuro de la educación, debemos hacer serias transformaciones en ese sistema caótico en el que estamos partiendo de la retroalimentación constante con nuestros alumnos y usándola como estrategia para ir mejorando y desarrollando el pensamiento complejo (Morin, 1999; Medina \& Deroncele, 2020b), a través de la educación a distancia. Es por ello que la OCDE (2016), propone a la evaluación formativa como retroalimentación y señala que dicho término deriva del campo de la ingeniería de sistemas; ya que es una información que genera impacto y ese mismo concepto es trasladado a la educación para mejorar los resultados educativos de los alumnos.

En esta misma perspectiva del involucramiento emocional de los estudiantes, Leighton (2019) afirma que se sabe poco sobre las interpretaciones que los estudiantes hacen en base a la retroalimentación, cómo la utilizan y cómo sacan conclusiones de sus propias autoevaluaciones. El docente es el que debe ayudar al proceso interpretativo del estudiante (Pellegrino, 2020). Sin embargo, hay poca investigación psicológica sistematizada sobre los estudiantes y las interpretaciones de la retroalimentación que hacen a través de modelos mentales. La interpretación de la retroalimentación requiere, según Leighton (2019). Entre tanto Huisman, Saab, Van Den Broek y Van Driel (2019) reafirman la importancia de la retroalimentación, pero esta vez orientada hacia la retroalimentación entre pares, es decir, de los mismos compañeros en el aula, la cual se define como la información que recibe un alumno a través de un compañero sobre las tareas relacionadas que realiza para mejorar su rendimiento académico. En este escenario, la retroalimentación es formativa porque mejora la habilidad y recomiendan que interactúen varios estudiantes y no solo con un compañero; ya que así se desarrolla mayor compromiso para la mejora del aprendizaje.

\section{CONCLUSIONES}

1. Es fundamental la reflexión del docente sobre su rol formador y como agente de cambio; el cual tiene la posibilidad de adecuar y adaptar de forma continua todos los procesos de evaluación según el contexto, necesidades e intereses del estudiante desplegando en su actuar, los principios de un aprendizaje por competencias.

2. La evaluación formativa en el contexto actual requiere cambiar las prácticas tradicionales ahora en aulas y espacios virtuales, así contribuir con la evolución de la enseñanza y aprendizaje en los espacios educativos a distancia; empleando diferentes recursos y aplicativos digitales para dinamizar los procesos de desarrollo de competencias que se proponen. Esto implica un compromiso de formación en las competencias digitales de los docentes para incorporar en su práctica pedagógica actual, así como concebir y reflexionar sobre los procesos de evaluación formativa que siempre deben considerar como fundamental en la valoración de los niveles de logro de los aprendizajes.

3. La aplicación de la evaluación formativa en el aula favorece el desempeño de los estudiantes considerando la retroalimentación reflexiva o por descubrimiento y descriptiva 
como estrategias para verificar los niveles de logro de competencias propuestas, que se evidencian mediante las producciones y actuaciones requeridas en cada experiencia de aprendizaje. Si en la realidad de la práctica educativa presencial, estos procesos evaluativos son una dificultad; entonces es momento de entender que si no se asumen cambios consistentes en el uso de estrategias, instrumentos, desarrollo de la planificación, análisis de las propuestas educativas seguirá las limitaciones de la función formativa de la evaluación y más aún en los espacios educativos a distancia donde la comunicación es más limitada por brechas económicas y sociales que obstaculizan la conectividad y acceso a los recursos virtuales.

4. En la evaluación formativa los estudiantes son participantes activos con sus docentes, entonces se debe valorar el desempeño individual y colectivo de los estudiantes quienes son copartícipes de su propia evaluación, ello implica la retroalimentación en función a las evidencias, así generar una transformación de la práctica evaluativa en la mejora de los aprendizajes con autonomía y reflexión. En los últimos años, se muestran evidencias donde señalan que la evaluación formativa mejora el desarrollo de las competencias las cuales están ligadas a las capacidades metacognitivas, mejora la calidad del aprendizaje y es perdurable en su vida. Con la evaluación formativa, se logra que los estudiantes sean activos, participativos con sus compañeros y profesores; ellos comparten sus propósitos de aprendizaje y comprenden cómo van progresando, qué es lo que necesitan aportar, cómo dar y mejorar en este proceso los estudiantes desarrollando sus habilidades de autoevaluación y coevaluación.

5. La retroalimentación es fundamental para el proceso de evaluación y constituye una estrategia clave en la evaluación formativa; pues el docente y el alumno necesitan interactuar de modo sincrónico y asincrónico; efectivizándose con ello el autoconocimiento y la autorregulación como pilares de transformación y de mejora continua. Aquí se destaca la importancia de reconfigurar el sentido ético de la evaluación, muy diferente al de un proceso inquisidor o que cause temor.

6. Mediante el análisis de las evidencias de los estudiantes en las actividades propuestas, el docente puede brindar la valoración del proceso de aprendizaje para tener con claridad los logros y dificultades en el desarrollo de los propósitos de aprendizaje; también tiene la posibilidad de hacer un análisis integral de los resultados para luego proponer su perfeccionamiento poniendo en práctica la retroalimentación reflexiva del aprendizaje de manera oportuna.

\section{REFERENCIAS}

Andrade, H. \& Brookhart, S. (2019). Classroom assessment as the co-regulation of learning. Assessment in Education: Principles, Policy \& Practice, 1-23. https://doi.org/10.1080/0969594X.2019.1571992

Bañuelos, A., \& Montero, G. (2017). La evaluación de la educación a distancia. Propuesta de una guía para la autoevaluación. Revista Hamut’ay, 4 (1), 31-44. http://dx.doi.org/10.21503/hamu.v4i1.1394

Barrón, M. C. (2020). La educación en línea. Transiciones y disrupciones. Educación y pandemia. Una visión académica. Universidad Nacional Autónoma de México; 66-74. http://132.248.192.241:8080/jspui/

bitstream/IISUE_UNAM/540/1/BarronC_2020_La_educacion_en_linea.pdf

Bizarro, W., Sucari, W., \& Quispe, A. (2019). Evaluación formativa en el marco del enfoque por competencias.

Revista

Innova Educación. 1 (3). https://revistainnovaeducacion.com/index.php/rie/article/view/45

Black, P., \& William, D. (1998) Assessment and Classroom Learning, Assessment in Education, 5, 7-71

Buenrostro, A. (2016). Programa de apoyo al aprendizaje escolar: Docencia, servicio e investigación. Revista Electrónica de Psicología de la FES-Zaragoza-UNAM, 6(11), 1-14. 
https://www.zaragoza.unam.mx/

wpcontent/Portal2015/publicaciones/revistas/rev_elec_psico/vol6_no1.pdf

Cuenca, R. y Vargas, J. (2018). Perú: el estado de políticas públicas docentes. Instituto de Estudios Peruanos, IEP. Diálogo Interamericano. https://repositorio.minedu.gob.pe/bitstream/handle/20.500.12799/5868/Per

$\%$ c3\%ba\%20el\%20estado\%20de\%20pol\%c3\%adticas\%20p\%c3\%bablicas\%20docentes.pdf? sequence $=1 \&$ isAllowed $=\mathrm{y}$

Cunill, M., \& Curbelo, L. (2021). An approach to self-regulation of learning through formative evaluation on medical education. Revista Educación Médica Superior. 35(1). http://scielo.sld.cu/scielo.php? script=sci_arttext\&pid=S0864-21412021000100019

Deroncele, A., Medina, P., \& Gross, R. (2020). Gestión de potencialidades formativas en la persona: reflexión epistémica y pautas metodológicas. Revista Universidad y Sociedad, 12(1), 97-104. https://rus.ucf.edu.cu/index.php/rus/article/view/1417

Deroncele, A., Nagamine, M., y Medina, D. (2020). Bases epistemológicas y metodológicas para el abordaje del pensamiento crítico en la educación peruana. Revista Inclusiones, 7(Número Especial), 6887.

http://

bkp.revistainclusiones.org/gallery/7\%20vol\%207\%20num\%20especialleabriljunio2020revinclusi.pdf

Díaz-Barriga, A. (2020). La escuela ausente., la necesidad de replantear su significado. Educación y pandemia. Una visión académica. Universidad Nacional Autónoma de México. 19-29. http://132.248.192.241:8080/xmlui/bitstream/handle/IISUE_UNAM/535/ DiazBarrigaA_2020_La_escuela_ausente_.pdf?sequence=1\&isAllowed $=\mathrm{y}$

Dorrego, E. (2016). Educación a distancia y evaluación del aprendizaje RED. Revista de Educación a

Distancia.

Núm. 50. Art. 12. 15-Jul-2016 DOI: http://dx.doi.org/10.6018/red/50/12 http://www.um.es/ead/red/50/ dorrego.pdf

Fraile, J., Sánchez, J., Alarcón, R., \& Ruiz, P. (2019). Documentos compartidos para la evaluación formativa en trabajos en grupo. Revista de Innovación y Buenas Prácticas Docentes, 8(1). https://doi.org/10.21071/ ripadoc.v8i1.11995

Huisman, B., Saab, N., Van Den Broek, P. \& Van Driel, J. (2019). $\square$ e impact of formative peer feedback on higher education students' academic writing: a Meta-Analysis. Assessment \& Evaluation in Higher Education, 44(6), 863-880. https://doi.org/10.1080/02602938.2018.1545896

Intriago, G. C., Carpio, D. A., Sobenis, J. A., \& Torres, R. J. (2020). Análisis de la evaluación formativa y su influencia en el aprendizaje virtual en la carrera de Educación Básica-UTB. 2020. Magazine de las $\begin{array}{lllll}\text { Ciencias: Revista de Investigación e Innovación, } & 5(7), & 4555 .\end{array}$ https://revistas.utb.edu.ec/index.php/magazine/article/view/920

Leighton, J. (2019). Students' Interpretation of Formative Assessment Feedback: $\square$ ree Claims for WhyWe Know So Little About Something So Important. Journal of Educational Measurement, 56(4), 793-814. https:// doi.org/10.1111/jedm.12237

Lezcano L. y Vilanova G. (2017). Instrumentos de evaluación de aprendizaje en entornos virtuales. Perspectiva de estudiantes y aportes de docentes. Universidad Nacional de la Patagonia Austral, Argentina. 9 (1); 1-36. https:// dialnet.unirioja.es/servlet/articulo?codigo $=5919087$

López, V. M., Sonlleva, M., \& Martínez, S. (2019). Evaluación formativa y compartida en educación. RIEE.

Revista

Iberoamericana de Evaluación Educativa. 12(1); 5-9. https://revistas.uam.es/riee/article/view/10844

Londoño, O., Maldonado Granados; L. F., \& Calderón, L. C. (2014). Guía para construir estados del arte. https:// iconk.org/docs/guiaea.pdf

López, S. (2017). Evaluación y aprendizaje. Marco ELE: Revista de Didáctica Español como Lengua Extranjera, (24), 
3. https://marcoele.com/descargas/24/fernandez-evaluacion_aprendizaje.pdf

Medina, P., \& Deroncele, A. (2019). La evaluación formativa desde el rol del docente reflexivo. Revista Maestro $\mathrm{y}$

Sociedad, 16(3); 597-610. http://www.maestroysociedad.uo.edu.cu

Medina, P., \& Deroncele, A. (2019b). La evaluación formativa desde el rol del docente reflexivo. Revista Maestro $\mathrm{y}$

Sociedad, 16(3), 597-610. http://www.maestroysociedad.uo.edu.cu

Minedu. (2016). Currículo Nacional de la Educación Básica. MINDEDU. http://www.minedu.gob.pe/curriculo/ $\mathrm{pdf} /$ curriculo-nacional-de-la-educacion-basica.pdf

Minedu. (2019). Planificación, mediación y evaluación de los aprendizajes en la Educación Secundaria. MINDEDU. https://repositorio.minedu.gob.pe/handle/20.500.12799/6646

Morales, C. R., \& Medina, P. (2020). Aprender a enseñar: un camino para el arquitecto docente. Revista Maestro y Sociedad, 17(3), 521-131. http://www.maestroysociedad.uo.edu.cu

Moreno, T. (2016). Evaluación del aprendizaje y para el aprendizaje Reinventar la evaluación en el aula (Primera ed). Universidad Autónoma Metropolitana.

Morin, E. (1999). La Cabeza Bien Puesta: Repensar la reforma, reformar el pensamiento. Ediciones Nueva Visión.

Organización para la Cooperación y el Desarrollo Económicos. (2016). Tendencias que Transforman la Educación. http://www.oecd.org/education/ceri/Trends-inEducation-2016-Executive-Summary-Spanish.pdf

Organización para la Cooperación y el Desarrollo Económicos. (2017). $\square$ e OECD Hadbook for Innovate Learning Enviroments. https://doi.org/10.1787/20769679

Pasek de Pinto, E., \& Mejía, M. T. (2017). Proceso general para la evaluación formativa del aprendizaje. Revista Iberoamericana de Evaluación Educativa. 10 (1); 177-193. https://revistas.uam.es/riee/article/view/7600

Pellegrino, J. (2020). Important Considerations for Assessment to Function in the Service of Education. Educational Measurement: Issues and Practice, 1-5. https://doi.org/10.1111/emip.12372

Pérez, Á., Hortigüela, D., Gutiérrez, C., \& Hernando, A. (2019). Andamiaje y evaluación formativa: Dos caras de la misma moneda. Revista Infancia, Educación y Aprendizaje (IEYA), 5(2), 559-565. http://revistas.uv.cl/ index.php/IEYA/index

Rodríguez, H., \& Salinas, M. (2020). La Evaluación para el Aprendizaje en la Educación Superior: Retos de la Alfabetización del Profesorado. Revista Iberoamericana de Evaluación Educativa, 13(1), 111-137. https:// doi.org/10.15366/riee2020.13.1.005

Segura, M. (2018). La función formativa de la evaluación en el trabajo escolar cotidiano. Revista Educación, 42(1); 20. https://doi.org/10.15517/revedu.v42i1.22743

Serra, J., García, J., Gil, P., Cejudo, C. y González, S. (2017). Estilos de aprendizaje y evaluación formativa: Estudio con universitarios de Educación Física chilenos. SPORT TK: Revista Euroamericana de Ciencias del Deporte.

7 (1). 129-138. https://digitum.um.es/digitum/bitstream/10201/57190/1/321991-1099211-1-SM.pdf

Valdivia, S., \& Fernández, M. (2020). La evaluación formativa en un contexto de renovación pedagógica: Prácticas al servicio del éxito. Revista Actualidades Investigativas en Educación, 20(1), 1-26. https://doi.org/10.15517/ aie.v20i1.40159

Vera, O. (2016). Como publicar artículos de revisión o actualización. Cuadernos Hospital de Clínicas, 57(3), 62-67. http://www.scielo.org.bo/scielo.php?script=sci_arttext\&pid=S1652-67762016000300009\&lng=es\&tlng=es

Villalaz, E. S., \& Medina, P. (2020). El currículo universitario peruano: aspectos complejos. Maestro y Sociedad, 7(1), 121-136. http://www.maestroysociedad.uo.edu.cu 